\title{
Selective laser trabeculoplasty in treating post-trabeculectomy advanced primary open-angle glaucoma
}

\author{
HONGYANG ZHANG ${ }^{1,2}$, YANGFAN YANG ${ }^{2}$, JIANGANG XU ${ }^{2}$ and MINBIN YU ${ }^{2}$ \\ ${ }^{1}$ Department of Ophthalmology, Guangdong General Hospital, Guangdong Academy of Medical Sciences, Guangzhou, \\ Guangdong 510086; ${ }^{2}$ State Key Laboratory of Ophthalmology, Zhongshan Ophthalmic Center, \\ Sun Yat-sen University, Guangzhou, Guangdong 510060, P.R. China
}

Received February 24, 2014; Accepted July 29, 2014

DOI: $10.3892 / \mathrm{etm} .2015 .2959$

\begin{abstract}
The aim of this study was to investigate the safety and efficacy of selective laser trabeculoplasty (SLT) treatment of patients with primary open-angle glaucoma (POAG) who could not obtain target intraocular pressure (IOP) through post-trabeculectomy medication. Sixteen patients with POAG (18 eyes), who could not obtain target IOP following medication and surgery, were treated with $360^{\circ} \mathrm{SLT}$. The IOP, anterior chamber inflammation, and daytime and long-term IOP fluctuations before and $2 \mathrm{~h}, 1$ day, 7 days, 1 month, 3 months, 6 months and 9 months after SLT were documented. SLT treatment success was defined as $>20 \%$ IOP reduction compared with the baseline IOP at 6 and 9 months after the laser treatment date. Prior to SLT, the patients were administered different types (average, $2.8 \pm 0.8$ ) of anti-glaucoma drugs and had an average IOP of $21.3 \pm 3.4 \mathrm{mmHg}$. Following SLT, the average IOP decreased to $16.2 \pm 3.0 \mathrm{mmHg}$ and the success rate was $77.7 \%$. The pre-SLT daytime IOP fluctuation was $4.1 \pm 1.4 \mathrm{mmHg}$, which decreased to $2.6 \pm 1.1 \mathrm{mmHg}$ following the laser treatment $(\mathrm{P}<0.05)$. In conclusion, this study demonstrated that SLT could reduce the IOP in post-trabeculectomy patients with POAG, and reduce the daytime IOP fluctuations.
\end{abstract}

\section{Introduction}

In 1983, Anderson and Parrish (1) found that specific optical radiation could damage pigmental structure. Theoretically, this effect could be applied to target tissues. In 1995, Latina and Park (2) applied this concept and were the first to conduct laser-selective treatment of the pigment-containing

Correspondence to: Professor Minbin Yu, State Key Laboratory of Ophthalmology, Zhongshan Ophthalmic Center, Sun Yat-sen University, 54 South Xianlie Road, Guangzhou, Guangdong 510060, P.R. China

E-mail: minbinyu@126.com

Key words: selective laser trabeculoplasty, target intraocular pressure, primary open-angle glaucoma, trabeculectomy trabecular meshwork. They showed that a specific wavelength laser could selectively hit the pigment-containing trabecular cells. Selective laser trabeculoplasty (SLT) uses the Q-switch doubling frequency $532 \mathrm{~nm}$ neodymium-doped yttrium aluminium garnet (Nd:YAG) laser with a pulse time of $3 \mathrm{nsec}$ and a diameter of $400 \mu \mathrm{m}$ to irradiate the trabecular meshwork. SLT selectively targets the pigmental trabecular cells, while the non-pigmental trabecular cells and the surrounding tissues are not affected by the laser energy. The high selectivity and extremely short laser pulse time can reduce the damage to the surrounding non-pigmented trabecular tissues. In a previous study, no coagulation due to thermal damage was observed in the tissues following SLT; however, pigment granules were disintegrated within trabecular cells and there was destruction of pigmental trabecular cells (3). By contrast, the surrounding cells and tissues that did not contain the pigments showed no changes. Therefore, SLT treatment is safe. In 2001, the Food and Drug Administration approved the clinical use of SLT and this provided a novel therapeutic approach for primary open-angle glaucoma (POAG) (4).

SLT has been widely used in clinical treatment since 2002 . Studies have shown that SLT can be used as one of the initial treatments of patients with POAG or in combination therapy when the maximum-tolerated medical therapy does not obtain satisfactory therapeutic effects (5-8). SLT can also be used as a therapeutic method to reduce the effective dose of anti-glaucoma drugs $(9,10)$. However, there have not been any reports on the use of SLT as a treatment of post-trabeculectomy patients with POAG.

Trabeculectomy is still considered the mainstay for medically uncontrolled glaucoma (11). Studies have shown that, even when anti-metabolic drugs are applied during surgery, the five-year success rate of trabeculectomy is $60-80 \%$ (12) and the 15 -year success rate is $52-59 \%(13,14)$. The postoperative filtering bleb scarring is the most important reason for surgical failure (15). The application of antimetabolites (such as mitomycin C) can reduce the scarring caused by filtering blebs and improve the surgical success rate, but certain patients remain who, due to a number of reasons, fail the surgeries. Trabeculectomy failure normally needs further laser or surgical intervention if the maximum medical therapy is insufficient. The difficulty of repeat trabeculectomy in these 
patients is significant. It is widely acknowledged that prior incisional surgery decreases the success rate of subsequent surgery for glaucoma (16). This is the most difficult issue in the treatment of glaucoma. For patients with advanced glaucoma whose target intraocular pressure (IOP) $(\leq 18 \mathrm{mmHg})$ cannot be achieved with filtering surgery and the administration of anti-glaucoma medications, the re-filtering surgery is a significant challenge for the patients and the physicians. SLT can reduce the IOP of patients with POAG, with no significant difference identified in the angle structure of these patients. Therefore, as a noninvasive treatment method, SLT provides a novel treatment option for patients with POAG who would normally require further IOP control following glaucoma surgery.

\section{Materials and methods}

Patients. Patients who were diagnosed with POAG and who underwent one or more trabeculectomies between May and December 2012 in the Zhongshan Ophthalmic Center, Sun Yat-sen University (Guangzhou, China) were selected for this study. Following the surgery, 16 patients (18 eyes) could not obtain the target IOP following the application of one or several anti-glaucoma drugs. This included 14 males (15 eyes) and two females (three eyes). The follow-up period was 6-9 months (Table I). This study was conducted in accordance with the Declaration of Helsinki and with approval from the Ethics Committee of Sun Yat-sen University. Written informed consent was obtained from all participants.

Inclusion criteria. Patients had to meet the diagnostic criteria of POAG established by the International Society of Geographical and Epidemiological Ophthalmology (17). As such, the patient had to i) have lost the majority of his/her vision with only a $5-10^{\circ}$ central or temporal vision island and have an eyeground exhibiting the typical depression of glaucomatous optic papilla and a cup/disc (C/D) area ratio of $\geq 0.8$, with a mean deviation of $<-12 \mathrm{~dB}$; ii) have undergone one or more trabecular surgeries, and been prescribed one or more anti-glaucoma drugs without obtaining the target IOP; iii) have a previous history without other ocular surgery; iv) have a previous history without diabetes and hypertension; v) not plan to become pregnant during the treatment and observation period; vi) be able to be followed-up on schedule; and vii) continue their medication for at least three months before SLT.

Exclusion criteria. The exclusion criteria were as follows: i) Other types of open-angle glaucoma; ii) achievement of the target IOP following the trabeculectomy; iii) the patient had previously undergone argon laser trabeculoplasty or other eye surgeries; iv) the other eye of the patient was blind; v) systemic or ocular disease requiring corticosteroid therapy; and vi) the patient was $<18$ years old.

Treatment termination indicator. If the intra-experimental IOP reached $30 \mathrm{mmHg}$ for $>4 \mathrm{~h}$, the IOPs of two post-treatment consecutive re-checks were higher than those prior to the treatment or serious complications occurred, the experiment was terminated.
Criteria for successful treatment. The treatment was considered to be successful if i) the IOP following the laser treatment was reduced by $>20 \%$ compared with the baseline IOP prior to the treatment, and ii) there were no serious complications.

Observation parameters and evaluation indicators. The best corrected visual acuity (BCVA) was determined with the international standard vision chart. Slit-lamp examination was performed by observing the cornea, anterior chamber depth, lens and vitreous body. The Goldman IOP was checked each time. The ultrasonic corneal pachymeter (DGH 1000; DGH Technology, Inc., Exton, PA, USA) was used to measure the central corneal thickness three times, and the average was calculated. IOP determination was performed using the Goldman applanation tonometer (AT $900 \mathrm{R}^{\circledR}$, Haag-Streit USA, Inc., Mason, OH, USA); the IOP was measured three times and the average IOP was calculated. The Goldman applanation tonometer (AT $900 \mathrm{R}^{\circledR}$,Haag-Streit USA, Inc. Mason, OH, USA) detection time-points were 8:00 a.m., 10:00 a.m., 12:00 a.m., 2:00 p.m. and 5:00 p.m. These detection time-points are referred to as IOP fluctuation during day time. The detection time-point for each measurement of IOP after the follow-up visit was 10:00 $\pm 1 \mathrm{~h}$ in the morning. Each measurement refers to the measurement of IOP for the follow up after 1, 3, 7 days and after 1 month. Daytime IOP curve tracing was performed by checking the daytime IOP curves prior to the treatment and those three, six and nine months after the treatment. The daytime IOP fluctuation was equal to the highest daytime IOP measured minus the minimum daytime IOP measured. The main postoperative complications were observed, the eyeground was examined by direct ophthalmoscopy and the C/D ratio was recorded.

SLT treatment. The $360^{\circ} \mathrm{SLT}$ treatment was performed by the same physician for all patients in this study. The Ellex SOLO ${ }^{\circledR}$ SLT Nd:YAG laser treatment apparatus (Ellex Medical Pty Ltd., Adelaide, Australia) was used. The doubling frequency Q-switch Nd:YAG laser had a single pulse of visible light, a wavelength of $532 \mathrm{~nm}$, a pulse width of $3 \mathrm{nsec}$, a facula spot diameter of $400 \mu \mathrm{m}$ and an energy range of 0.3-2.6 mJ. The initial energy of the laser was set to $0.8 \mathrm{~mJ}$, with $0.1 \mathrm{~mJ}$ as the amplitude value when increasing or decreasing the laser energy. When the bubbles formed, the laser energy was reduced by $0.1 \mathrm{~mJ}$ for the treatment. The single and non-repeated laser spot treatment was performed towards the trabecular meshwork along the nasal or temporal side. The treatments in each quadrant were performed 25 times, with a $360^{\circ}$ chamber angle.

Statistical analysis. The SPSS 18.0 statistical package (SPSS, Inc., Chicago, IL, USA) was used to analyze the data for significance. The continuous variables with normal distribution were assessed using the bilateral Student's t-test or t-matching test, while the variables that did not meet the normal distribution were analyzed using the Mann-Whitney U test.

\section{Results}

The preoperative age, IOP, BCVA, refraction, corneal thickness and $\mathrm{C} / \mathrm{D}$ ratio are shown in Table $\mathrm{I}$. The average 
Table I. Basic information for the patients post-trabeculectomy but pre-selective laser trabeculoplasty.

\begin{tabular}{lc}
\hline Parameter & Value \\
\hline Age, years & $37.5 \pm 11.2(18-64)$ \\
Preoperative IOP, $\mathrm{mmHg}$ & $21.3 \pm 3.4(17-32)$ \\
Corneal thickness, $\mu \mathrm{m}$ & $527.1 \pm 27.1(485-568)$ \\
Refraction, D & $-2.8 \pm 2.1(0--7.0)$ \\
Preoperative medication types, $\mathrm{n}$ & $2.8 \pm 0.8(2-4)$ \\
BCVA & $0.3 \pm 0.3(0.4-1.0)$ \\
Cup/disc ratio & $0.86 \pm 0.10(0.8-0.9)$ \\
Initial energy $(\mathrm{mJ})$ & $0.6 \pm 0.1(0.4-0.7)$ \\
Treatment energy $(\mathrm{mJ})$ & $60.9 \pm 11.6(50-83)$ \\
\hline
\end{tabular}

Unless otherwise stated, results are presented as the mean $\pm \mathrm{SD}$ (range). BCVA, best corrected visual acuity; IOP, intraocular pressure; $\mathrm{SD}$, standard deviation.

number of trabecular surgeries received by all the patients was $1.7 \pm 0.5$ (range, 1-3). The post-glaucoma surgery time was $2.4 \pm 1.1$ years and the average follow-up time was 6.3 months.

Preoperative medication. The average number of preoperative medications prescribed per patient was $2.8 \pm 0.8$. A total of $22.2 \%$ of the patients used four anti-glaucoma eye drop medications, $38.9 \%$ of the patients used three anti-glaucoma eye drop medications and $38.9 \%$ of the patients used two anti-glaucoma eye drop medications.

IOP. The preoperative IOP in this patient population ranged between 17 and $32 \mathrm{mmHg}$, with the average at $21.3 \pm 3.4 \mathrm{mmHg}$. The postoperative 2 -h IOP ranged between 12 and $27 \mathrm{mmHg}$, with the average at $17.8 \pm 4.0 \mathrm{mmHg}$. The postoperative one-day IOP was $8-24 \mathrm{mmHg}$, with the average at $14.2 \pm 3.9 \mathrm{mmHg}$. The postoperative seven-day IOP was $12-22 \mathrm{mmHg}$, with the average at $16.5 \pm 2.8 \mathrm{mmHg}$. The postoperative one-month IOP was $11-20 \mathrm{mmHg}$, with the average at $15.5 \pm 2.5 \mathrm{mmHg}$. The postoperative three-month IOP ranged between 10 and $24 \mathrm{mmHg}$, with the average at $15.9 \pm 3.1 \mathrm{mmHg}$. The postoperative six-month IOP ranged between 11 and $26 \mathrm{mmHg}$, with the average at $16.9 \pm 3.9 \mathrm{mmHg}$. The postoperative nine-month IOP ranged between 11 and $19 \mathrm{mmHg}$, with the average at $16.2 \pm 3.0 \mathrm{mmHg}$. The IOP time curve is shown in Fig. 1. The postoperative IOP decreased significantly when compared with the preoperative IOP $(\mathrm{t}=5.820, \mathrm{P}<0.001)$. The IOP of all the patients prior to SLT was $>15 \mathrm{mmHg}$ and the IOP of $77.8 \%$ of the patients was $>18 \mathrm{mmHg}$, with the average follow-up period of 6.3 months. The IOP of $27.8 \%$ of the patients was $<15 \mathrm{mmHg}$, and the patients with an IOP $>18 \mathrm{mmHg}$ saw a reduction in their IOP by $16.7 \%$. The changes in IOP distribution prior and subsequent to SLT are shown in Fig. 2. Three patients failed the treatment, one patient was prescribed anti-glaucoma medication, and two patients received the second anti-glaucoma surgery.
Table II. Post-selective laser trabeculoplasty adverse reactions.

\begin{tabular}{lc}
\hline Adverse reaction & $\mathrm{n}(\%)$ \\
\hline Transient ocular hypertension & $0(0.0)$ \\
Pink eye & $10(55.6)$ \\
Fuzzy vision & $6(33.3)$ \\
Mild eye pain & $4(22.2)$ \\
\hline
\end{tabular}

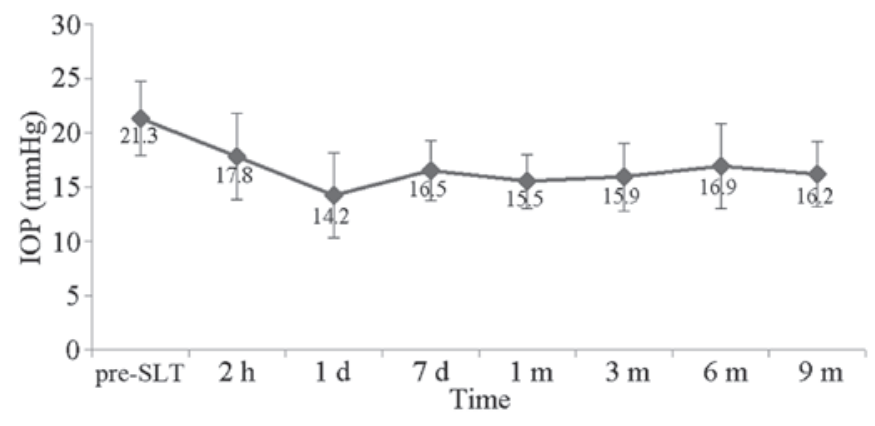

Figure 1. Changes in IOP prior and subsequent to SLT. IOP, intraocular pressure; SLT, selective laser trabeculoplasty.

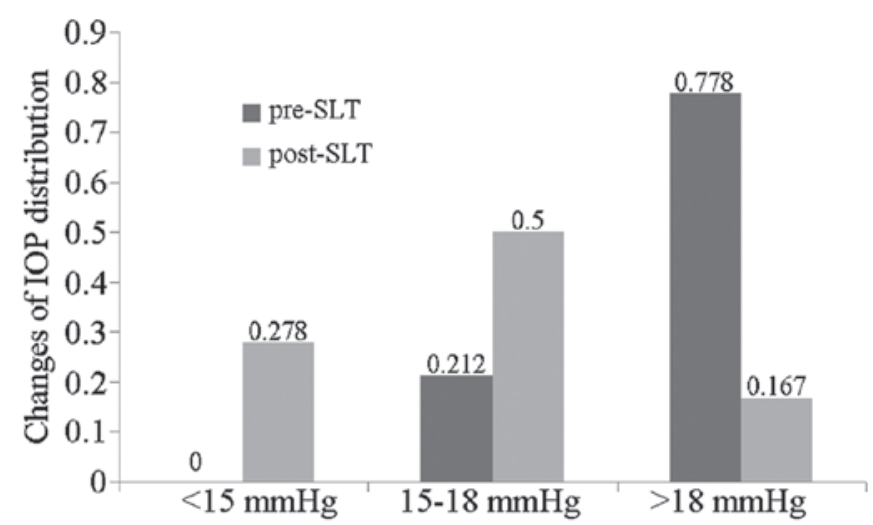

Figure 2. Changes in IOP distribution prior and subsequent to SLT. IOP, intraocular pressure; SLT, selective laser trabeculoplasty.

Success rate. The reduction in IOP in all the patients $(100 \%)$ was $>20 \%$ one day after the treatment. In the last follow-up, $77.7 \%$ of the patients had a reduction in their IOP of $\geq 20 \%$.

Effect of SLT on IOP fluctuation. The average IOP fluctuation prior to SLT was $4.1 \pm 1.4 \mathrm{mmHg}$, and the postoperative IOP fluctuation was $2.6 \pm 1.1 \mathrm{mmHg}(\mathrm{t}=3.424, \mathrm{P}=0.003)$.

Adverse reactions. The most common postoperative adverse reactions were mild anterior chamber inflammation, mild eye pain, fuzzy vision and pink eye, which returned to normal 24-48 $\mathrm{h}$ after the procedure. None of the patients appeared to have transient ocular hypertension. The gonioscopy was performed in the late follow-up and revealed no formation of peripheral anterior synechia (Table II). 


\section{Discussion}

Clinically, glaucoma treatment has focused on reducing IOP. It has been recognized that reducing IOP to normal levels is insufficient in the control of IOP in patients with advanced glaucoma (18). The different disease course of glaucoma and the different degrees of optic nerve damage can result in a different tolerance of retinal ganglion cells and lamina cribrosa towards IOP. Therefore, the target IOP specific to each patient with glaucoma must be determined. The concept of a target IOP is not only dependent upon the 'individual tolerance pressure', but is also determined by the threshold pressure. The threshold pressure is the IOP under which there would be no further damage to the glaucomatous optic nerve during treatment or follow-up. Under this IOP, the loss rate of retinal ganglion cells would not be greater than that induced by age, and the optic neuropathy may be decelerated or even terminated. Practice has proven that IOP reduction can effectively control the damage to the visual field in glaucomatous patients, and can delay the progression speed of glaucomatous optic neuropathy. Furthermore, fewer fluctuations in IOP can reduce the glaucomatous visual impairment. Patients with advanced glaucoma require reduced IOP to protect the already-damaged optic nerves $(19,20)$.

The tolerance of optic nerves in patients with advanced glaucoma towards IOP is significantly decreased and, in order to prevent further glaucomatous damage, it is best to reduce the IOP to $<15 \mathrm{mmHg}(21,22)$. All the patients in this study were patients with advanced POAG, and the average post-glaucoma surgery time was 2.4 years. The patients had received anti-glaucoma surgery 1.7 times, on average, and the average number of anti-glaucoma medications received by each patient was 2.8 . The average preoperative IOP was $21.3 \mathrm{mmHg}$. During the final post-SLT follow-up, the mean postoperative IOP decreased to $16.2 \mathrm{mmHg}$, with the success rate at $77.7 \%$. In this study, the IOP of all the patients was $>15 \mathrm{mmHg}$ prior to SLT, and the IOP of $77.8 \%$ of the patients was $>18 \mathrm{mmHg}$. Following SLT, the IOP of $27.8 \%$ of the patients was $<15 \mathrm{mmHg}$, and $83.3 \%$ of the patients had an IOP of $\leq 18 \mathrm{mmHg}$. The average reduction amplitude of SLT in the patients with advanced POAG that had received the filtering surgery was $\sim 5.1 \mathrm{mmHg}$, which was the same as the therapeutic results of those who had not received the filtering surgery (4). A previous study showed that each $1 \mathrm{mmHg}$ reduction in IOP in patients with advanced glaucoma reduced their vision loss by $10 \%(18,23)$. Studies of advanced glaucoma therapy have also shown that the level of IOP is positively correlated with the visual field damage $(24,25)$. During the six-year follow-up, the patients with an average IOP of $<18 \mathrm{mmHg}$ had little or no vision loss, while those with an IOP $>18 \mathrm{mmHg}$ exhibited clear and progressive vision damage. The SLT decreased the IOP of $83.3 \%$ of the patients to $<18 \mathrm{mmHg}(22,23)$. Therefore, in patients with advanced glaucoma, SLT can further reduce the IOP of the patient based on the application of anti-glaucoma drugs, helping the patient achieve or approach the target IOP. This would be an important factor in the protection of the optic nerve in patients with advanced glaucoma.

During the IOP reduction in the patients with advanced POAG, it is also necessary to further reduce the IOP fluc- tuations. The normal IOP fluctuation range is 3-6 $\mathrm{mmHg}$, while the IOP fluctuation of patients with POAG would be significantly higher, 2-3-fold that of the IOP fluctuation in the normal population. In patients with POAG, the IOP fluctuation should be strictly controlled, and a number of studies have emphasized the importance of regulating these fluctuations $(24,25)$. In the present study, SLT could not only further reduce the IOP of the patients, which could not be achieved through post-trabeculectomy medication, but it could also reduce the post-trabeculectomy IOP fluctuation in patients with advanced glaucoma. Kóthy et al (26) reported that SLT could reduce the daytime IOP fluctuation of patients with POAG. Nouri-Mahdavi et al (27) performed the Advanced Glaucoma Intervention Study (AGIS) and found through regression analysis that the IOP fluctuation (daytime or intra-follow-up) could predict the glaucomatous visual field progression. IOP fluctuations were more important than the average IOP, and only when the IOP fluctuation was excluded was the average IOP value meaningful. Every additional $1 \mathrm{mmHg}$ in IOP fluctuation [standard deviation (SD)] resulted in a 30\% increased risk of visual field progression. The five-year observation results of the AGIS showed that the long-term IOP fluctuation of $\leq 3 \mathrm{mmHg}$ (SD) caused significant visual field progression, while the IOP fluctuation of $<3 \mathrm{mmHg}$ helped to reduce the visual field progression. The patients with a long-term IOP fluctuation of $<3.1 \mathrm{mmHg}$ were found to have a 2.89 -fold greater risk of visual field progression than patients with a $2 \mathrm{mmHg}$ fluctuation (28). The study by Hong et al (29) in 2007 revealed that an IOP fluctuation of $>2 \mathrm{mmHg}$ would also increase the risk of visual field damage progression, even if the IOP was $<18 \mathrm{mmHg}$. An IOP fluctuation of $<2 \mathrm{mmHg}$ would be better able to prevent the vision damage. Thus, in the treatment of advanced glaucoma, one would not only need to control the IOP at levels below the target IOP, but also reduce the glaucomatous visual field damage caused by the IOP fluctuation.

In the present study, when patients received the post-trabeculectomy SLT, the average IOP fluctuation was reduced from the preoperative value of $4.1 \mathrm{mmHg}$ to the postoperative value of $2.6 \mathrm{mmHg}$, and this difference was significant $(\mathrm{P}<0.05)$. The results revealed that SLT could not only reduce the post-trabeculectomy mean IOP in patients with advanced glaucoma, but could also control the daytime IOP fluctuation. In glaucomatous patients with advanced visual field changes, the reduction in IOP fluctuation would exhibit positive effects towards the protection of the visual function of the patient.

With regard to the three patients who failed the treatment, two received additional anti-glaucoma drugs and one underwent filtering surgery treatment. All the patients exhibited an IOP reduction, with the greatest reduction observed one day after the surgery. None of the patients exhibited serious complications, such as transient high IOP, peripheral anterior synechia or uveitis.

The chamber angular structure of the patients with POAG was not changed following the trabeculectomy, which provided the conditions for the SLT treatment. In terms of the validity of SLT as a method to further reduce IOP following trabeculectomy, our study showed that SLT safely, effectively and easily reduced the IOP, with few side-effects, and provided a viable alternative for treating patients with advanced POAG. 
The follow-up of these patients should be further extended, and a control study should also be conducted to observe the effects of SLT on IOP fluctuations.

\section{References}

1. Anderson RR and Parrish JA: Selective photothermolysis: precise microsurgery by selective absorption of pulsed radiation. Science 220: 524-527, 1983.

2. Latina MA and Park C: Selective targeting of trabecular meshwork cells: in vitro studies of pulsed and CW laser interactions. Exp Eye Res 60: 359-371, 1995.

3. Kramer TR and Noecker RJ: Comparison of the morphologic changes after selective laser trabeculoplasty and argon laser trabeculoplasty in human eye bank eyes. Ophthalmology 108: 773-779, 2001.

4. Realini T: Selective laser trabeculoplasty: a review. J Glaucoma 17: 497-502, 2008

5. Hirn C, Zweifel SA, Töteberg-Harms M and Funk J: Effectiveness of selective laser trabeculoplasty in patients with insufficient control of intraocular pressure despite maximum tolerated medical therapy. Ophthalmologe 109: 683-690, 2012 (In German).

6. Koucheki B and Hashemi H: Selective laser trabeculoplasty in the treatment of open-angle glaucoma. J Glaucoma 21: 65-70, 2012.

7. Klamann MK, Maier AK, Gonnermann J and Ruokonen PC: Adverse effects and short-term results after selective laser trabeculoplasty. J Glaucoma 23: 105-108, 2014.

8. Bovell AM, Damji KF, Hodge WG, Rock WJ, Buhrmann RR and Pan YI: Long term effects on the lowering of intraocular pressure: selective laser or argon laser trabeculoplasty? Can J Ophthalmol 46: 408-413, 2011.

9. Francis BA, Ianchulev T, Schofield JK and Minckler DS: Selective laser trabeculoplasty as a replacement for medical therapy in open-angle glaucoma. Am J Ophthalmol 140: 524-525, 2005.

10. Abdelrahman AM and Eltanamly RM: Selective laser trabeculoplasty in Egyptian patients with primary open-angle glaucoma. Middle East Afr J Ophthalmol 19: 299-303, 2012.

11. Wells AP, Bunce C and Khaw PT: Flap and suture manipulation after trabeculectomy with adjustable sutures: titration of flow and intraocular pressure in guarded filtration surgery. J Glaucoma 13: 400-406, 2004

12. Law SK, Shih K, Tran DH, Coleman AL and Caprioli J: Long-term outcomes of repeat vs initial trabeculectomy in open-angle glaucoma. Am J Ophthalmol 148: 685-695, 2009.

13. Suzuki R, Dickens CJ, Iwach AG, Hoskins HD Jr Hetherington J Jr, Juster RP, Wong PC, Klufas MT, Leong CJ and Nguyen N: Long-term follow-up of initially successful trabeculectomy with 5-fluorouracil injections. Ophthalmology 109: 1921-1924, 2002.

14. Chen TC, Wilensky JT and Viana MA: Long-term follow-up of initially successful trabeculectomy. Ophthalmology 104: 1120-1125, 1997.

15. Picht $G$ and Grehn F. Classification of filtering blebs in trabeculectomy:biomicroscopy adn functionality. Curr Opin Ophthalmol 9: 2-8, 1998.
16. Olali C, Rotchford AP and King AJ. Outcome of repeat trabeculectomies. Clin Experiment Ophthalmol 39: 658-664, 2011.

17. Foster PJ, Buhrmann R, Quigley HA and Johnson GJ. The definition and classification of glaucoma in prevalence surveys. Br J Ophthalmol 86: 238-242, 2002.

18. Leske MC, Heijl A, Hussein M, Bengtsson B, Hyman L, Komaroff E; Early Manifest Glaucome Trial Group. Factors for glaucoma progression and the effect of treatment: the early manifest glaucoma trial. Arch Ophthalmol 121: 48-56, 2003.

19. Hong S, Seong GJ and Hong YJ: Long-term intraocular pressure fluctuation and progressive visual field deterioration in patients with glaucoma and low intraocular pressures after a triple procedure. Arch Ophthalmol 125: 1010-1013, 2007.

20. Inatani $M$, Iwao $K$, Inoue $T$, Awai M, Muto $T$, Koga $T$, Ogata-Iwao M, Hara R, Futa R, Tanihara H: Long-term relationship between intraocular pressure and visual field loss in primary open-angle glaucoma. J Glaucoma 17: 275-279, 2008.

21. Shirakashi M, Iwata K, Sawaguchi S, Abe H and Nanba K. Intraocular pressure-dependent progression of visual field loss in advanced primary open-angle glaucoma: a 15-year follow-up. Ophthalmologica 207: 1-5, 1993.

22. No authors listed. The Advance Glaucoma Intervention Study (AGIS): 7. The relationship between control of intraocular pressure and visual field deterioration. AGIS Investigators. Am J Ophthalmol 130: 429-440, 2000.

23. AGIS Investigators: The Advanced Glaucoma Intervention Study (AGIS): 12. Baseline risk factors for sustained loss of visual field and visual acuity in patients with advanced glaucoma. Am J Ophthalmol 134: 499-512, 2002.

24. Konstas AG, Hollo G, Astakhov YS, Teus MA, Akopov EL, Jenkins JN and Stewart WC: Factors associated with long-term progression or stability in exfoliation glaucoma. Arch Ophthalmol 122: 29-33, 2004.

25. Heijl A, Leske MC, Bengtsson B, Bengtsson B and Hussein M; Early Manifest Glaucoma Trial Group: Measuring visual field progression in the Early Manifest Glaucoma Trial. Acta Ophthalmol Scand 81: 286-293, 2003.

26. Kóthy P, Tóth M and Holló G: Influence of selective laser trabeculoplasty on 24-hour diurnal intraocular pressure fluctuation in primary open-angle glaucoma: a pilot study. Ophthalmic Surg Lasers Imaging 41: 342-347, 2010.

27. Nouri-Mahdavi K, Hoffman D, Coleman AL, Liu G, Li G, Gaasterland D and Caprioli J; Advanced Glaucoma Intervention Study: Predictive factors for glaucomatous visual field progression in the Advanced Glaucoma Intervention Study. Ophthalmology 111: 1627-1635, 2004.

28. Asrani S, Zeimer R, Wilensky J, Gieser D, Vitale S and Lindenmuth K: Large diurnal fluctuations in intraocular pressure are an independent risk factor in patients with glaucoma. J Glaucoma 9: 134-142, 2000.

29. Hong S, Seong GJ and Hong YJ: Long-term intraocular pressure fluctuation and progressive visual field deterioration in patients with glaucoma and low intraocular pressures after a triple procedure. Arch Ophthalmol 125: 1010-1013, 2007. 